\title{
Pressure measurement in oesophageal varices: preliminary report on a new non-invasive method
}

\author{
PH GERTSCH AND J-J MEISTER* \\ From the Service de Chirurgie B CHUV, Lausanne, Switzerland \\ ${ }^{*}$ Fondation Pour le Progres de l'Homme
}

SUMmaRY Pressure in oesophageal varices has been measured either by using an invasive puncture technique, or a non-invasive pneumatic pressure sensor. Experience with these techniques and their limitations prompted a new way of measuring pressure in oesophageal varices. This was done by observing through a transparent plastic balloon fixed to the tip of a gastroscope the pressure equilibrium between the varix and the balloon. One hundred measurements on an experimental model showed the reliability of the system. Measurements on eight patients with the balloon followed immediately by the puncture technique, showed good correlation.

Treatment of bleeding oesophageal varices has been and still is a subject of major concern. Sclerotheraphy has reduced the mortality of the first incident of bleeding but the mortality rate is still around $50 \%$ among patients in the Child's $\mathrm{C}$ category.'

The search for new criteria to define the place of prophylactic sclerotherapy, or other forms of preventive treatment is therefore important. Measurement of pressure in oesophageal varices has been until recently an unexploited source of information. Among the proposed techniques, needle puncture of the varix is technically the simplest and probably the most reliable $;{ }^{23}$ but is invasive with potential risk of haemorrhage. A non-invasive technique was described in 1982 and evaluated on patients under narcosis ${ }^{45}$ its value, however, seems questionable if used during routine endoscopy under simple sedation. ${ }^{6}$ Here we present the preliminary results of a new non-invasive technique which was tested on an experimental model and evaluated on a small number of patients.

\section{Methods}

PATIENTS

The principle of this method is to detect the minimum pressure necessary to collapse the varix wall while directly observing it. A balloon made of a nonexpansile transparent polyethylene sheet $(10 \times 12 \mathrm{~cm})$

Address for correspondence: Ph Gertsch, Viszerale Chirurgie, Inselspital Bern, Switzerland.

Received for publication 26 January 1987. folded in two, rounded off at one extremity and sealed with a domestic hot electric wire, is fixed at the tip of a gastrofibroscope (Olympus GIF Q - 10) (Fig. 1 ). The balloon is inflated until it starts collapsing the oesophageal varices, using a $50 \mathrm{cc}$ syringe connected to a plastic catheter ( $2 \mathrm{~mm}$ diameter) introduced through the biopsy channel of the endoscope into the balloon. The space between the biopsy channel and the plastic tube is sealed at the extremity of the endoscope to ensure no air leak. The balloon has a diameter of $3.5 \mathrm{~cm}$, which is bigger than that of the oesophagus so that full inflation will not occur. The pressure measured in the balloon will therefore correspond to that produced on its external wall.

The pressure in the system is measured by an electronic manometer (Statham element) connected to the plastic catheter which terminates in the balloon. Pressure measured with the electronic

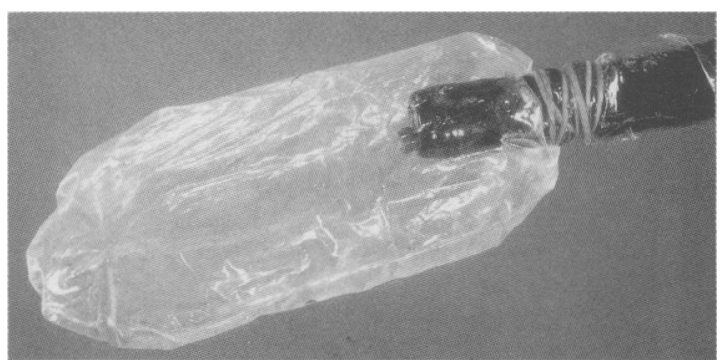

Fig. 1 Varices are observed through a partially inflated transparent and non-expansile balloon fixed at the tip of a gastrofibroscope. 


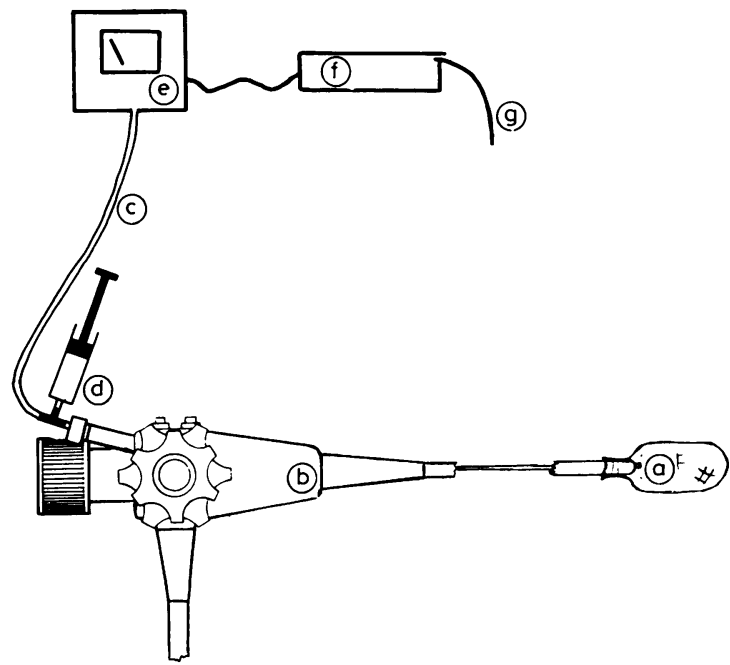

Fig. 2 Diagram of the measuring system: $a=$ balloon; $b=$ gastrofibroscope $; c=$ plastic catheter $d=50$ cc syringe; $e=$ electronic manometer $;=$ recorder $; g=$ foot-operated switch.

manometer is recorded on millimetric paper (Fig. 2). Measurements were taken on an experimental model consisting of a surgically removed saphenous vein fixed on the interior wall of a plastic tube $2.5 \mathrm{~cm}$ in diameter, connected to a water column whose height was varied, producing various pressures in the vein (Fig. 3). The endoscope with the deflated balloon was introduced into the plastic tube. The balloon was then inflated with air using the $50 \mathrm{ml}$ syringe, to the point where the vein started to collapse. Pressure in the balloon was recorded at the moment of collapse (zero $=$ atmospheric pressure).

The method was then tested on eight patients before sclerotherapy. Pressure zero was defined as the atmospheric pressure in the balloon system. The endoscope with the deflated balloon was introduced down to the cardia. The balloon was then slightly inflated and the endoscope withdrawn to about $3 \mathrm{~cm}$ above the cardia. Varices were observed through the transparent wall of the balloon during its gentle and slow inflation until the point where they started to collapse. Pressure was recorded at this precise moment on millimetric paper. The endoscope was then withdrawn and a therapeutic endoscope (Olympus GIF T - 10) introduced into the oesophagus. Intravariceal pressure was measured by puncture using a slow perfused system $(0 \cdot 2 \mathrm{cc} / \mathrm{min})$ as previously described. ${ }^{3}$ Pressure zero was defined as the atmospheric pressure and determined by gauging the zero when holding the endoscopic puncture needle at the estimated level of the lower oesophagus before introduction of the endoscope. Pressure measurements were recorded on millimetric paper.
Results obtained in the experimental model and 'in vivo' were analysed using a linear regression test.

\section{Results}

Measurements on the experimental model were easy to carry out and no particular training was needed to determine the pressure. The 100 recorded pressure measurements could be read precisely on the millimetric paper. The comparison with the corresponding heights of the water column showed a good correlation $(r=0.904, p<0.0001)$ (Fig. 4). Variations were present and increased directly with the measured pressure, thus reflecting a constant percentage error. The size of varices of the patients submitted to sclerotherapy were, according to Paquet's classification ${ }^{7}$ of stage $3(n=6)$ and $4(n=2)$. The endoscope with the deflated balloon was introduced without difficulty; gentle inflation of the balloon $3 \mathrm{~cm}$ above the cardia allowed a clear vision of the varices through the balloon wall (Fig. 5). Determination of the pressure was easy in all cases. Measurements of intravariceal pressure by puncture were done without any significant problem immediately before sclerotherapy. Bleeding after withdrawal of the needle was present in all cases and ceased spontaneously.

The measurements taken with the two techniques showed a good correlation $(\mathrm{r}=0.961, \mathrm{p}<0.0001)$ (Fig. 6).

\section{Discussion}

Measurement of pressure in oesophageal varices could give valuable information for future studies on portal hypertension. Published data regarding the relationship between the size of oesophageal varices, the portal pressure, and the risk of haemorrhage are still controversial. ${ }^{-110}$ Direct measurement of the pressure in oesophageal varices has so far been done by few teams and has remained in the state of research.

Our experience with different techniques available lead us to believe that the perfused puncture technique is at present time the most reliable $;{ }^{236}$ because the slow infusion of liquid, paravenous insertion of the needle is easily detected by a constant pressure rise on the recorded curve. The puncture of small varices (Paquet's stage 1 and 2) is, however, sometimes more difficult or even impossible. Although bleeding after withdrawal of the needle usually ceases spontaneously, the use of this technique will probably be limited in most centres to patients who undergo sclerotherapy during the same endoscopy. Transient bacteriaemia during sclerotherapy has been clearly established" and could be another 


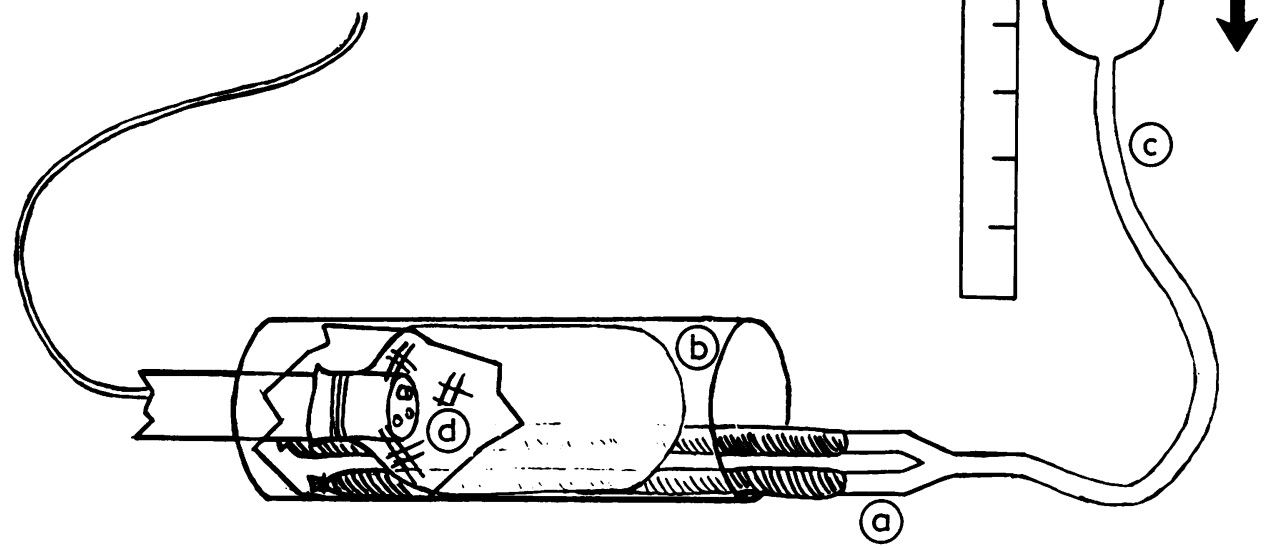

Fig. 3 Experimental model: a surgically removed saphenous vein (a) was fixed on the inner wall of a plastic tube (b) and connected to a water column (c); the endoscope with the balloon (d) was introduced into the plastic tube for measurements.

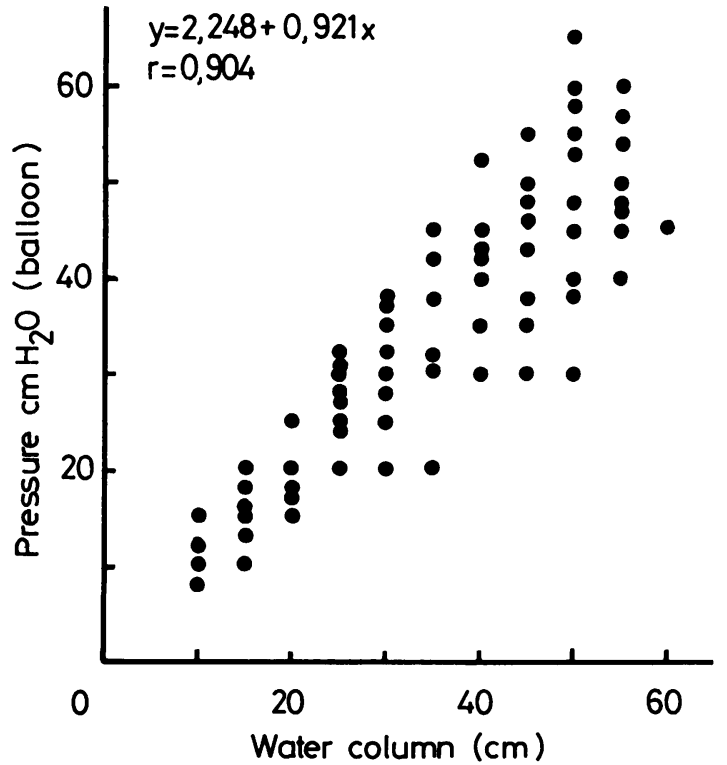

Fig. 4 Comparison of measurements carried out on the experimental model showing a good correlation between the height of the water column and the pressure measured with the balloon $(r=0.904, p<0.0001)$. argument against unrestricted use of the puncture technique which might have the same drawback.

A non-invasive method for measuring intravariceal pressure was described in $1982 .{ }^{4}$ Although we considered initial results promising when measurements were done under narcosis,${ }^{45}$ measurements during routine endoscopy with simple sedation did not give similar results. Ideal positioning of the pressure sensor on varices proved to be difficult, because of the peristaltis of the oesophagus, retching and coughing movements, and constant swallowing which obscured the endoscopic view. Under these conditions, discrimination between pressure recording and artefacts was difficult and the results were therefore often unreliable. ${ }^{6}$

Although further development of the endoscopic pressure sensor is foreseen, a new method was investigated. The use of a balloon to measure the pressure in oesophageal varices by compressing them and determining the pressure equilibrium between the balloon and the blood vessel is in principle similar to the wide and longstanding use of the cuff arterial pressure measurement. This technique has several advantages over the other non-invasive method. Measurement is done with direct and clear vision of the varices as secretions and even blood are swept 


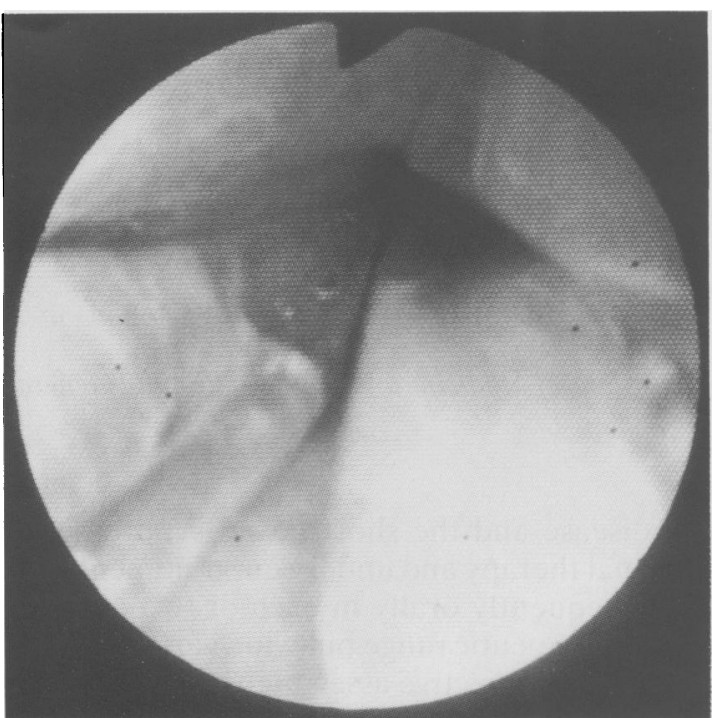

Fig. 5 Oesophageal varices observed through the balloon before pressure was applied to collapse them.

away by the balloon. Furthermore measurement of the pressure in bleeding oesophageal varices might even be possible with this method.

Comparison of the pressures measured by balloon and puncture technique could be confusing if pressure differed in different varices on the same patient; apparently this is not the case. ${ }^{2}$

Our results show the feasibility of such measure-

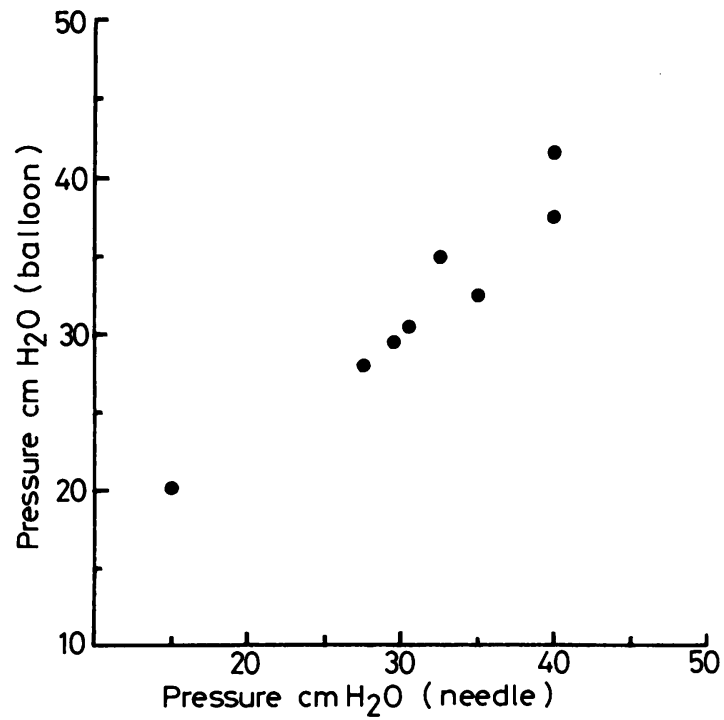

Fig. 6 Comparison of measurements carried out on 8 patients with the needle and with the balloon technique showing a good correlation ( $r=0.961, p<0.0001)$. ments whose real precision remains to be determined with increasing experience. Improvements in the measuring device, especially by replacement of the syringe with an easier to control inflating system, should allow more precise determination of the pressure balance between the varix and the balloon. The value of the method, however, remains to be determined for measurement of pressure in small sized varices and for assessment of small variations in pressure.

We believe that this simple technique deserves further interest; in its actual form, its realisation does not need any particular engineering. The balloon technique could be easily adapted and available anywhere.

We would like to thank Mr G Corazzola, from the Division Autonome d'Electronique Medicale, CHUV, for technical assistance.

\section{References}

1 The Copenhagen Esophageal Varices Sclerotherapy Project. Sclerotherapy after first variceal hemorrhage in cirrhosis. A randomized multicenter trial. $N$ Engl J Med 1984; 311: 1594-600.

2 Staritz M, Poralla T, Meyer zum Buchsenfelde KH. Intravascular oesophageal variceal pressure (IOVP) assessed by endoscopic fine needle puncture under basal conditions, Valsalva's manoeuvre and after glyceryl trinitrate application. Gut 1985; 26: 525-30.

3 De Renck M, Burette A, Van Gossum M, et al. Endoscopic measurement of the pressure in the oesophageal varices of cirrhotic patients. Correlation with portal pressure. Acta Endoscopica 1984; 14: 121-7.

4 Mosimann R. Nonaggressive assessment of portal hypertension using endoscopic measurement of variceal pressure. Am J Surg 1982; 143: 212-4.

5 Gertsch Ph, Bohnet J, Mosimann R. Endoscopic nonaggressive assessment of oesophageal variceal pressure compared with wedged hepatic venous pressure in alcoholic liver cirrhosis. Endoscopy 1983; 15: 101-3.

6 Staritz M, Gertsch Ph. Pressure measurements in oesophageal varices: first comparison of two techniques. Endoscopy 1986; 18: 253-4.

7 Paquet KJ. Prophylactic endoscopic sclerosing treatment of the esophageal wall in varices. A prospective controlled randomized trial. Endoscopy 1982; 14: 4-5.

8 Gertsch $\mathrm{Ph}$, Loup $\mathrm{P}$, Diserens $\mathrm{H}$, Mosimann F, Mosimann R. Endoscopic noninvasive manometry of esophageal varices: prognostic significance. Am J Surg 1982; 144: 528-30.

9 Groszmann RJ. Reassessing portal venous pressure measurements. Gastroenterology 1984; 86: 1611-7.

10 Conn HO. The varix volcano connection. Gastroenterology 1980; 79: 1333-7.

11 Sauerbruch T, Holl J, Ruckdeschel G, Forstl J, Weinzierl M. Bacteriaemia associated with endoscopic sclerotherapy of oesophageal varices. Endoscopy 1985 ; 17: $170-2$. 